Nouvelles perspectives en sciences sociales

Revue internationale de systémique complexe et d'études relationnelles

\title{
Le Fardeau de la légèreté : une étude de Kimchi d'Ook Chung
}

\section{Ziyan Yang}

Volume 8, numéro 2, mai 2013

Sur le thème de représentations identitaires et expressions culturelles de la francophonie canadienne à travers ses pratiques artistiques et médiatiques

URI : https://id.erudit.org/iderudit/1016471ar

DOI : https://doi.org/10.7202/1016471ar

Aller au sommaire du numéro

\section{Éditeur(s)}

Prise de parole

\section{ISSN}

1712-8307 (imprimé)

1918-7475 (numérique)

Découvrir la revue

Citer cet article

Yang, Z. (2013). Le Fardeau de la légèreté : une étude de Kimchi d’Ook Chung. Nouvelles perspectives en sciences sociales, 8(2), 85-100.

https://doi.org/10.7202/1016471ar
Résumé de l'article

Depuis la naissance de la littérature du Québec qui vise la construction de l'identité québécoise, le rapport entre la saisie du Soi et la perception de l'Autre constitue un noyau dur du discours littéraire. Aujourd'hui, avec l'émergence des écrivains migrants ou néo-québécois (différenciés des écrivains québécois " de souche "), le Soi et l'Autre se rapprochent à tel point que leurs frontières se dissolvent dans cette écriture dite " migrante ». Cet article s'intéresse à la représentation de l'identité dans le premier roman Kimchi par Ook Chung, un écrivain québécois d'origine asiatique. En nous appuyant sur les recherches de Charles Taylor et de Paul Ricoeur portant sur la notion de l'identité, nous réfléchissons d'abord sur la dialectique entre le Soi et l'Autre dans la quête identitaire du protagoniste. Ensuite, nous analysons le langage de Chung à la croisée des cultures selon le concept de l'« hybridité » proposé par Homi Bhabha. Finalement, nous portons notre attention au rôle de l'art et de l'écriture dans l'élaboration identitaire chez Chung. 


\title{
Le Fardeau de la légèreté : une étude de Kimchi d'Ook Chung
}

\author{
ZIYAN YANG \\ Dalhousie University, Halifax
}

\section{Introduction}

l'heure actuelle où la mondialisation popularise et banalise

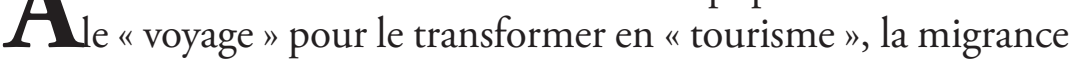
devient un lieu commun et la diaspora se développe avec le nombre croissant d'individus ayant une identité à trait d'union. Dans le cas du Québec, les vagues d'immigration, l'acte du multiculturalisme et surtout le dynamisme de ceux qui vivent et écrivent leur migration, font croître l'intérêt pour l'apport des Néo-québécois au paysage littéraire ${ }^{1}$. Par leur questionnement

Cette écriture pousse les critiques à proposer pour elle de nombreuses appellations dont écriture " ethnique ", " transculturelle ", " néo-québécoise ", " postcoloniale », " mineure " ou " multiculturelle ». Parmi ces termes, celui d' "écriture migrante " élaboré par Robert Berrouët-Oriol et Robert Fournier paraît le mieux prémuni contre une catégorisation fossilisante basée sur l'origine nationale et ethnique tout en abordant les problématiques majeures telles que l'exil, l'hybridité et l'identité (Robert Berrouët-Oriol et Robert Fournier, "L'Émergence des écritures migrantes et métisses au Québec ", Québec Studies, $\mathrm{n}^{\circ}$ 14, 1992, p. 7-22). Toutefois, ce terme reste également problématique en milieu de la critique littéraire. Simon Harel, par exemple, a signalé dans Les Passages obligés de l'écriture migrante la naïveté de la louange facile de l'"identité migrante ", qui malgré la mise en relief de la diversité culturelle, risque de contribuer «à un nouveau "patriotisme" interculturel où le Québec [occupe] une place de choix " (Simon Harel, Les Passages obligés de l'écriture migrante, Montréal, XYZ, 2005, p. 69). 
identitaire et leur imaginaire métis, ces écrivains venus d'ailleurs reflètent la "crise d'identité " collective ${ }^{2}$. À l'heure où chacun peut prétendre être métis ${ }^{3}$ et avoir mille identités culturelles différentes $^{4}$, la réflexion des Néo-québécois sur la migration, les phénomènes du déracinement et de l'enracinement, l'échange et le métissage, permet de redéfinir la notion d'identité dès lors caractérisée par la pluralité et l'instabilité.

Nous proposons ici une étude de l'identité chez Ook Chung, un écrivain néo-québécois aux déracinements multiples. Né au Japon de parents coréens, Ook Chung a immigré au Québec à un jeune âge. Il a fait ses études à Montréal et en France. Après un recueil de nouvelles intitulé Nouvelles orientales et désorientées, et où se développe l'opposition entre l'Orient et l'Occident, Ook Chung publie son premier roman Kimchi ${ }^{5}$, dont le narrateur

$\overline{2}$ Nous avons recours à la notion de l'identité que Charles Taylor propose dans Les sources du moi. Il la situe dans un cadre éthique où le sujet peut " essayer de juger cas par cas ce qui est bien ou valable ", de " prendre position " par rapport au Bien (Charles Taylor, Les Sources du moi, Montréal, Boréal, 1998, p. 46), si bien que l'essence de notre identité « se confond (au moins partiellement) avec le positionnement que nous adoptons par rapport à cette orientation [vers le Bien] " (Charles Taylor, op. cit., p. 54-55). À l'axe éthique, Taylor ajoute l'autre axe important pour « la pleine définition de l'identité d'un individu ", soit " une référence à une communauté qui détermine [sa position par rapport aux questions morales et spirituelles]" (Charles Taylor, op. cit., p. 57). Cette " communauté " renvoie à ce que Taylor appelle "réseau d'interlocution " qui regroupe des "interlocuteurs " liés au sujet par le langage. Mais ce qui caractérise la société contemporaine, c'est l'absence d'un cadre de référence partagé par tous, suite au déclin des croyances religieuses et à l'essor de l'individualisme. Il s'agit d'une " crise d'identité ", dans laquelle les choses échouent à prendre une signification stable et les possibilités de vie ne font plus l'objet d'une évaluation cohérente. Dans le cas des immigrants, cette crise d'identité se vit de façon encore plus intense à cause de leur changement total de la culture et du " réseau d'interlocution".

3 Selon Anne Ancelin Schützenberger, nous sommes tous des métis dans la mesure où nous sommes nés du métissage d'au moins deux familles différentes, celle de notre mère et celle de notre père (Anne Ancelin Schützenberger Aïe, mes ä̈eux!, Paris, Desclée de Brouwer, 1993, p. 101).

4 Nancy Huston, dans "Le Déclin de l' "identité"? ", a montré combien la société moderne, notamment par la voie des médias, a élargi les horizons du lecteur à l'infini et en a donc pulvérisé l'identité culturelle (Nancy Huston, "Le Déclin de l' "identité"? ", Âmes et corps : textes choisis 1981-2003, Montréal, Leméac, 2004, p. 57-77).

5 Ook Chung, Kimchi, Paris, Le Serpent à plumes, 2001, 246 p. 
protagoniste ressemble à l'auteur par sa carrière littéraire et sa nature de "canadien-francophone-né-au-Japon-de-parentscoréens ». Ce récit réunit des anecdotes et les souvenirs d'un certain $\mathrm{O}$. Kim qui est à la recherche de ses origines : son enfance innocente dans une famille pleine de violence et de mensonges, son adolescence douloureuse à cause de l'exclusion raciste dont il fut victime au Québec, ses voyages dans ses pays d'origine, ses premières amours ainsi que sa découverte de l'identité de son père biologique et de l'existence de sa propre fille. Ce roman d'apprentissage présente une véritable quête identitaire, et nous amène à réfléchir sur le rôle de l'origine, de la patrie, de la filiation et de la création dans la formation de l'identité.

Afin d'aborder le concept de l'identité, des philosophes, des anthropologues, des sociologues et des psychologues ont tous proposé des approches différentes. Nous choisissons de nous appuyer sur celle de Paul Ricœur qui, dans Soi-même comme un autre, base son analyse de l'identité sur la dialectique entre le Soi et l'Autre. Si nous effectuons un tel choix, c'est non seulement parce que ce texte de Ricœur, avec des études sur le plan d'énonciation, de narration et d'éthique, répond pertinemment au besoin de la critique littéraire, mais aussi parce que cette approche, en présentant la formation de l'identité comme un perpétuel dynamisme entre l'altérité et la "mêmeté ", perçoit l'identité plutôt comme un projet que comme un produit figé. Paul Ricœur distingue deux usages majeurs du concept de l'identité : d'une part, l' «identité-mêmeté » ou l'« identité-idem » englobe une série de significations, y compris l'unicité, l'identitésimilitude, la continuité dans le temps et la permanence dans le temps ${ }^{6}$; d'autre part, l' "identité-ipséité » ou l'« identité-ipse » "n'implique aucune assertion concernant un prétendu noyau non changeant de la personnalité ${ }^{7}$ » et peut se constituer de toute altérité. C'est " la dialectique du soi et de l'autre que $s o i^{8}$ " qui maintient l'élaboration de l'identité comme une visée. Cette

Paul Ricœur, "L'Identité narrative ", Esprit, n ${ }^{\text {os }}$ 7-8, 1988, p. 296.

Paul Ricœur, Soi-même comme un autre, Paris, Seuil, 1990, p. 13.

Ibid., p. 13. 
dialectique nous guidera dans notre étude portant en premier lieu sur la quête d'appartenance qu'entreprend le narrateur, espace de migrance où la légèreté de la non-appartenance met l'identité à mort. Une même dialectique caractérise également l'imaginaire d'Ook Chung, cette fois à la manière " hybride " en termes d'Homi Bhabha. En effet, dans Les Lieux de la culture, Bhabha envisage l'hybridité culturelle comme un "tiers espace" " entre les deux polarités du Soi et de l'Autre, lieu où se situe la culture, comme une vision et un projet plutôt que comme une entité unitaire et prédéterminée. C'est à travers le dynamisme qui mène l'identité au-delà de sa fixité vers ce tiers espace que se réalise l'hybridité culturelle. L'écriture de Chung, à la croisée des formes d'art et des sources culturelles semble s'inscrire dans ce lieu marqué par une mobilité cosmopolite, ce qui dans un deuxième temps fera l'objet de notre analyse. Finalement, nous réfléchirons à la manière dont l'art, et surtout l'écriture, peut servir de remède au déracinement dont souffrent les " mal placés " chez Chung. Si nous centrons notre réflexion sur une figure du poids en résumant chaque partie par un rapport dialectique entre la légèreté et la lourdeur, c'est pour compléter notre étude avec une conception de l'identité basée sur la culture sinisée : Shenfen, identité en chinois et racine d'où dérive l'équivalent japonais, signifie littéralement le poids corporel.

\section{Légèreté de la migrance, fardeau de l'identité aliénée}

Si l'émigration est un prélude à la renaissance pour beaucoup qui aspirent à un recommencement de la vie, Chung associe pour sa part ce déplacement au meurtre identitaire d'O. Kim. À cause de l'émigration de ses parents, son identité a d'abord dû être dédoublée : lors de leur départ pour l'Amérique, ses parents ont truqué sa date de naissance pour obtenir une réduction sur leurs billets; à l'arrivée à Montréal, son nom coréen a remplacé son ancien nom japonais. Plus tard, pourtant, cette double identité est mise à mort par l'exclusion subie à l'école secondaire. Sans

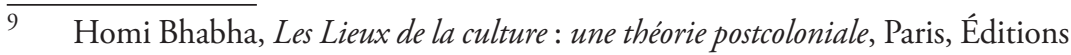
Payot et Rivages, 2007, p. 80. 
cesse ridiculisé par ses camarades de classe à cause de son nom étranger, de l'épicanthus de ses yeux, et somme toute de ses traits asiatiques, Kim intériorise le rejet de sa propre différence, se considère lui-même comme étranger et se décrit comme "un noyé à la dérive, un noyé ne se sachant pas noyé ${ }^{10}$ ". En posant un regard rétrospectif sur ces années de souffrance, le narrateur décrit cette perte identitaire ainsi :

Il me semble parfois que j'ai été un cadavre noyé pendant plus de vingt ans de ma vie, mais un cadavre obstiné qui refuserait de couler, tout bouffi et gorgé d'eau saline, à moitié décomposé déjà, ballotté au gré des courants océaniques dans un labyrinthe de vagues, à la recherche d'un port où ressusciter ${ }^{11}$.

À l'encontre du discours exaltant l'harmonie et la richesse prometteuse du métissage, l'enfance mal enterrée de Kim dévoile la migrance libre comme lieu de souffrance pesante. La liberté qui permet à l'individu de dépasser les frontières physiques, crée également les conditions d'une non-appartenance, d'une perte de repères et même d'une aliénation et d'une inertie existentielles. Avec le départ et le changement d'entourage, l'unicité et la permanence du moi subit d'atroces épreuves dans la mesure où l'individu doit acquérir de nouvelles langues et de nouveaux habitus culturels. Mais le moi du passé dont cette rupture n'a pu se débarrasser pèse sur le personnage comme un cadavre, à tel point qu'il explique le pneumothorax dont il souffre par l'existence d' " un foetus gelé en lui, enroulé tel en serpent autour de son poumon gauche ", "foetus de l'écolier qu'[il] avai[t] été, à l'époque où [il] subissai[t] les brimades de [s]es condisciples, à l'époque où quelque chose était mort en [lui $]^{12}$."

Portant ce foetus qu'est son passé mal enterré, Kim entrevoit dans le voyage, et surtout celui vers son continent natal, une possibilité d'acquérir le sens de l'orientation. Comme le précise Charles Taylor dans Les Sources du moi: "Afin de nous faire une idée de celui que nous sommes, nous devons avoir quelque idée de la façon dont nous le sommes devenus et de la

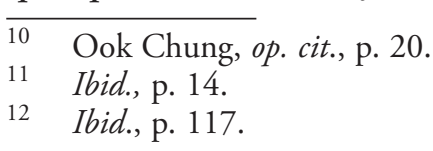


direction que nous prenons ${ }^{13}$. " Kim, après avoir été longtemps en proie à la cadavérisation identitaire, s'engage dans la voie qui le conduit vers ses origines d'abord par la lecture et l'écriture, sortes de voyages imaginaires, puis par des voyages véritables. Dans ce sens, les périples de Kim, avec ses aventures et ses mésaventures, ses pertes et ses gains, forment un roman d'apprentissage. Cependant, comme nous allons découvrir, il s'agit plutôt d'un roman d'apprentissage à rebours, ou d'un roman de désapprentissage, car la maturation du héros suit un cheminement vers l'origine et vise sa réconciliation avec son identité d'origine, une partie de son Moi rendue Autre à cause de son immigration.

Soumis à des souffrances indicibles à l'école, Kim comble ses heures de vagabondage entre cette "zone toxique " et la maison par la lecture. À ce plaisir, s'ajoute celui de l'écriture, autre moyen d'évasion ${ }^{14}$. Grâce à cet acte de création dont nous traiterons le rôle un peu plus tard, son imaginaire depuis longtemps colonisé par les "blancs " commence à réclamer son continent d'origine oublié, si bien qu'il commence à écrire " pour voyager en imaginaire en des pays fraternels ${ }^{15}$ ». Mais ce sont les vrais voyages qui lui permettent de retrouver son origine, ses racines et cette identité perdue.

Depuis son arrivée en Amérique et au contact de l'image des «blancs », O. Kim intériorise toute dévalorisation de sa différence physique par son entourage " blanc » et considère cette différence comme une altérité à rejeter. Son imaginaire ainsi " colonisé " évite toute attirance des traits asiatiques, " comme si entre [lui et les autres Asiatiques] existe un tabou incestueux ${ }^{16}$. Cependant, il éprouve un jour au Mexique une sensation de bonheur à se fondre dans un entourage de têtes noires. Ainsi se réveille son désir pour la similitude et sa prise de conscience de ce qui fait sa mêmeté. Cette réconciliation avec sa propre différence physique

13 Charles Taylor, op. cit., p. 71.

14 Kim écrit des poèmes qui témoignent de sa désorientation identitaire et s'imagine une vie heureuse au Canada dans le but de la raconter à ses cousins résidant au Japon.

15 Ook Chung, op. cit., p. 21.

16 Ibid., p. 20. 
déclenche chez lui une forte attirance pour la beauté physique asiatique : il se sent poussé vers le quartier chinois de toutes les villes qu'il visite, et est ému à la simple vue d'un visage asiatique dans la foule.

Incapable de situer son appartenance culturelle tant sur le plan géographique (Québec, Japon, ou Corée) que sur le plan idéologique (Occident ou Orient), Kim souligne le dynamisme récurrent entre le Soi et l'Autre qui régit sa quête d'appartenance culturelle et qui empêche toute appartenance à une culture ou à un pays monolithique et unitaire : il se sent spirituellement chez lui dans un groupe d'étudiants francophiles qui partagent son goût pour les écrivains japonais suicidaires; il reconquiert au Japon son identité coréenne par la découverte de son attachement irrévocable à kimchi, mets traditionnel coréen. Au cours d'un bref voyage dans le pays de ses ancêtres, bien qu'il n'y connaisse ni la langue ni les habitants, il s'enivre de la familiarité des inconnus fraternels ainsi que de la beauté de la chanson Arirang, hymne folklorique des Coréens, qui évoque d'ailleurs l'exil éternel et les départs sans retours ${ }^{17}$. Chaque fois que le narrateur croit avoir trouvé la pièce manquante pour résoudre le " puzzle " de son identité, une reconfiguration le déstabilise et le remet dans un état d'inachèvement. Par contre, il réalise que l'identité, loin d'être prédéfinie comme un "puzzle ", s'apparente à un projet en perpétuelle construction et déconstruction, un projet qui s'oriente vers l'accomplissement sans jamais y arriver.

C'est lors d'une visite à Paris à la recherche de son premier amour que Kim découvre dans les catacombes grâce à une lettre de son père biologique sa véritable origine familiale, tout en apprenant la fragilité de toute racine : exilé coréen écrivant en japonais, dont le nom Kim Chi-Hee renvoie par la sonorité à son point de repère identitaire kimchi, son père biologique l'encourage à " chérir son déracinement " et à "s s'attacher à une foi, à un dieu, plutôt qu'à un pays ", car "la recherche des racines

\footnotetext{
$17 \quad$ Lauteur explique dans une note de bas de page l'histoire que raconte Arirang, celle d'un jeune homme qui quitte sa famille tout en sachant qu'il ne reviendra sans doute plus jamais.
} 
comme panacée est une illusion ${ }^{18}$. Toute quête d'appartenance spécifique s'avérant vaine ou du moins insuffisante, Kim perçoit son périple comme une espèce d'effacement existentiel à reculons, ou, pour emprunter les termes de Ching Selao, une sorte de "dénaissance pour la renaissance ${ }^{19}$ " à la manière taoïste ${ }^{20}$. Ce " noyé à la dérive » sur lequel pèse le poids de l'ancienne identité morte, se sent soudain "purgé de toute rancœur " en revenant à son lieu de naissance. Il trouve le véritable but de son odyssée, soit de " mieux revenir en [lui]-même " et de "respirer cette expansion de l'âme ${ }^{21}$ ». La migrance, en tant que lieu de rencontre d'hétérogénéités, constitue en ce sens pour le sujet migrant un espace lui permettant de se connaître par l'Autre et par la différence, lui permettant de forger et bricoler, dans la dialectique entre la mêmeté et l'altérité, sa propre identité. Grâce à l'élan qui pousse les deux termes de ce rapport dialectique à se rejoindre, se tisse un espace d'» hybridité ", où fleurit un imaginaire d'» ambivalence " à la croisée des cultures, pour emprunter les termes de Bhabha.

\section{Une mobilité sans frontières, un bagage culturel lourd}

Contrairement aux Nouvelles orientales et désorientées, premier ouvrage de Chung où se déploie une "imagination tordue " ${ }^{22}$, Kimchi se rapproche du vraisemblable et de la tendresse sincère sans pourtant renoncer à "l'héritage culturel mondial ${ }^{23}$ ".

18 Ook Chung, op. cit., p. 236.

19 Ching Selao, "Écriture butô et altérité : Kimchi d'Ook Chung ", dans Janusz Przychodzen (dir.), Asie du soi, Asie de l'autre, Québec, Les Presses de l'Université Laval, 2009, p. 66.

20 En effet, le retour à l'état d'avant-naissance, pour les taoïstes, évoque le retour à l'Origine perdue. Dans cet état originel et idéal, tous les êtres se fusionnent avec le Dao, Voie ultime de l'univers qui engendre tous les êtres.

21 Ook Chung, op. cit., p. 14-15.

22 Dans ce recueil de nouvelles, l'auteur nous transporte de l'Inde au Canada, en passant, entre autres, par le Japon, la Corée, l'Argentine et la France. Il représente des êtres hors du commun, des " monstres de solitude " vivant dans des contextes culturels très divers. Cette collection d'êtres marginaux témoigne d'un sens de la différence nourri sans doute par l'héritage multiple de l'auteur.

23 Tina Mouneimne-Wojtas, "L'Imaginaire "asiatique” de Ying Chen, d'Ook 
Au niveau lexical, le récit est parsemé de mots anglais, japonais et coréens, faisant écho à l'aveu moqueur du narrateur, ce "polyglotte dysfonctionnel » selon lequel ne sortent de sa bouche que « des brochettes d'expressions tronquées en japonais, coréen, anglais, toutes confondues ${ }^{24}$ sans qu'il puisse même finir une phrase dans la même langue. Tout en créant un exotisme sonore, ce champ lexical hybride englobe bien des mots lourds de connotations culturelles différentes, mais liées par une sorte d'» alchimie " littéraire, comme le prouve cette réflexion sur son prénom :

Dans le symbole du yin et du yang qui décore le drapeau de mon pays ancestral, tous les éléments sont reliés. Le feu transforme l'eau en nuage, le nuage dans le ciel se transforme en pluie, la pluie se cristallise en neige, et le cycle recommence. Dans mon prénom, il y a le symbole chinois $\mathrm{du}$ feu, mais il y a aussi, dans son épellation occidentale, la lettre " $\mathrm{O}$ ", qui rime avec eau... O comme " Orient », comme " Occident " 25

L'idéogramme chinois renvoyant au feu et la lettre rimant avec " eau » en français impliquent la lutte fusionnelle entre les deux pôles (oriental et occidental) apparemment incompatibles chez le narrateur : celui-ci avoue dès le début du roman que "ces symboles conflictuels ou tonifiants, [sont] [...] à l'image de l'Orient et l'Occident qui luttent en [lui] depuis toujours ${ }^{26}$. Quant au cycle figuratif des éléments symboliques, on y distingue également par un "télescopage linguistique ${ }^{27}$ " la référence aux deux personnages qui marquent la quête identitaire du narrateur : sa fille naturelle Yuki, soit " neige " en japonais, et son élève amérasienne dont le nom, Amy, veut dire " pluie " en japonais $(A m e)$. Amy, qui souffre de discrimination au Japon à cause de son physique occidental, constitue pour Kim un miroir où il se reconnaît, un Autre du Soi. Honteuse de ses traits occidentaux,

Chung et d'Aki Shimazaki ", dans L'Imaginaire du roman québécois contemporain : actes du colloque Université Masaryk de Brno, Brno, Masarykova univerzita Brno, Montréal, Université du Québec à Montréal, Figura, Centre de recherche sur le texte et l'imaginaire, 2006, p. 143.

24 Ook Chung, op. cit., p. 64.

25 Ibid, p. 246.

26 Ibid., p. 17.

27 Tina Mouneimne-Wojtas, op. cit., p. 144. 
Amy ssenferme chez elle et évite toute socialisation. Aux yeux du narrateur, Amy est physiquement «l'Ennemi au visage blanc qui avait saccagé [s] on adolescence "; lui, est "l'Ennemi d'Amy ", "celui qui la ridiculisait en classe en la traitant de sale gaiji ${ }^{28}{ }^{29}$. Ces deux êtres apparaissent comme des reflets inversés, devant le miroir d'un destin qui tenterait, par le pardon et la compréhension, de réaliser " une justice symétrique ${ }^{30}$ ». Quant à Yuki, la fille de Kim née de son premier amour, elle est pour lui un membre de famille étranger, une fille naturelle ignorée qui le rattache à son territoire d'origine. C'est en compagnie de cette jeune fille japonaise que le narrateur retourne au Chinatown de Yokohama, son lieu de naissance où il, se retrouvant au point de départ de sa vie, connaît enfin sa résurrection identitaire. Dans cette petite citation sur le prénom du narrateur, les référents d'origines culturelles multiples se juxtaposent et s'interpénètrent, de sorte que se dessine un espace qui n'appartient à aucune des cultures mobilisées ou, selon les termes d'Homi Bhabha, un espace d'» hybridité ". Par un jeu de va-et-vient, l'auteur se positionne dans cet espace interstitiel entre différentes cultures à la source de son inspiration, ce qui lui offre un horizon symbolique enrichissant pour envisager la relation entre la culture et la langue dans la formation identitaire.

Cet imaginaire métis est également créé par les innombrables citations qui émaillent la narration, allant de la peintre américaine Romaine Brooks à Van Gogh, de l'écrivain chinois Lu Xun aux écrivains japonais de Burai-ha (soit les Décadents), en passant par de nombreuses critiques littéraires, des dictons coréens et japonais ainsi que des inscriptions relevées dans les catacombes de Paris. Cette riche intertextualité témoigne d'un imaginaire nourri par plusieurs formes d'art du monde entier, y compris le cinéma (surtout japonais et coréen), la peinture, l'architecture,

\footnotetext{
28 Gaijin : mot japonais pour étranger/étrangère.

29 Ook Chung, op. cit., p.139-140.

30 Cette affinité est si forte que l'affection d'Amy pour Kim ressemble pour la première à de l'inceste : en effet, la peinture de deux lunes jumelles et celle qui s'intitule Inceste karmique réalisées par la jeune artiste Amy nous suggèrent que cet amour secret est " incestueux " à ses yeux.
} 
la musique classique occidentale, les chants traditionnels orientaux et le Butô, danse japonaise à laquelle l'auteur a consacré une section entière. Cette abolition des frontières physiques et virtuelles donne naissance à un imaginaire « migrant » qui se reflète notamment dans la représentation de l'étudiante japonaise Hiroé, incarnation du premier amour du narrateur.

D’emblée, Kim est gagné par son charme "déséquilibré » : une grâce délicate accompagnée d'une expression de "chien battu " et d'une dentition abîmée. Ce charme à la frontière entre la beauté et la difformité s'affirme avec une figure de la ruine. Effectivement, l'image des ruines, souvent en contraste avec la beauté d'une jeune femme, sert à accentuer la beauté face au temps et à appeler l'empathie envers l'éphémère dans la poétique classique japonaise ${ }^{31}$. Sous la plume de Chung, la belle Hiroé partage avec le narrateur un goût pour Dazai, un écrivain japonais décadent et hanté par la désillusion de l'après-guerre. Lors de son premier tête-à-tête avec Kim, Hiroé, en badinant, lui lance une invitation au suicide à l'exemple de Dazai, et la reprend sur un pont en lui proposant de sauter ensemble. Affalée sur les genoux

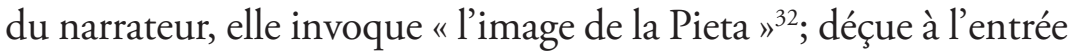
d'un temple à Kyoto, elle lui rappelle "le tableau La Derelitta " ${ }^{33}$. Selon Kim, elle est une "naguru no onna" (fille violente et destructrice à la japonaise) dont les mots favoris sont « déprimée " et " triste "; sa beauté ressemble à celle des jeunes filles en fleurs décrites par Proust mais se lie inextricablement à l'ivresse à cause de leurs rendez-vous réguliers au bar; discrètement fidèle à la foi chrétienne comme à la pratique de la musique, elle ressemble pour Kim aux religieux évoqués par l'écrivain chrétien Endo Shusaku et dissimule sa propre musique avec une réserve orientale. Ce syncrétisme de figures de la ruine, qui puise autant dans la culture orientale qu'occidentale, met au jour un être "d'abîme ", qui appelle l'Autre qu'est le narrateur, victime solitaire attirée par la mort. La figure de l'abîme, avec la plongée inévitable dans la

\footnotetext{
31 Il s'agit du concept esthétique et spirituel «Mono no aware » qui se traduit littéralement par "l'empathie envers les choses".

32 Ook Chung, op. cit., p. 49.

33 Ibid., p. 90.
} 
profondeur, évoque également une mort symbolique suivie d'une renaissance et d'une résurrection, dans le sens bouddhiste et chrétien tant pour l'un que pour l'autre ${ }^{34}$. Grâce à cet entremêlement d'éléments hétérogènes d'origines métisses aux niveaux linguistique, imaginaire et symbolique, se forme un espace riche à la frontière tant des cultures que des formes d'art. La migrance pleine de légèreté qu'effectue l'auteur dans cet espace permet au roman de nouer des liens étroits avec toutes les cultures et toutes les formes d'art. Elle met ainsi en œuvre un riche métissage textuel, soulignant la tension entre une identité de plus en plus fluide, et le positionnement délicat des écrivains migrants.

\section{Écriture : entre la légèreté et la solidité}

Parallèlement à son voyage en Asie pour « chercher le dépaysement et aller au-delà de l'altérité35 ", l'exploration artistique de Kim lui offre de nouvelles possibilités salutaires pour canaliser la mort, à la fois impasse et "force vitale dévoyée " ${ }^{36}$.

Si l'écriture est, selon Nancy Huston, le seul pays des « écrivains divisés " (c'est-à-dire de ceux qui vivent et écrivent plus d'une culture), cette "nation en imagination » est plutôt un leurre pour le narrateur écrivain de Kimchi. En effet, Kim avoue qu'il est "venu à l'écriture par suite d'une certaine infirmité face à la vie " causée par la mort identitaire datant de son adolescence. Cependant, cet " écrivain accidentel " pour qui l'écriture est " une sorte de déversoir émotif, de salut psychologique et peut-être aussi spirituel ${ }^{37}$ " se voit transformé en un "infirme à vie ${ }^{38}$ » en perpétuel déséquilibre, car "l'écriture est un précipité de la vie ou de la mort, elle aide à vivre comme elle tue parfois, on dirait

34 Notons qu'après sa rupture avec Kim, Hiroé coupe tout contact avec sa famille et ses amis pour s'installer dans un pensionnat chrétien à Paris. Kim, lui, après une longue quête identitaire, découvre le message de son père biologique qui l'invite à " chérir le déracinement ", et regagne l'innocence de son enfance avec sa fille retrouvée.

35 Jules Nadeau, "Ook Chung : la vie est un match de sumo ", La Presse, 14 septembre 2003, p. F2.

36 Ook Chung, op. cit., p. 43.

37 Ibid., p. 59.

38 Ibid., p. 156. 
qu'il n'y a pas de milieu entre ces extrêmes. ${ }^{39}{ }_{\text {» }}$ Cet équilibre constant entre le confort et la souffrance qui fait la raison d'être de l'écrivain et qui ne fait que perpétuer sa situation de naufragé, s'explique plus clairement dans la lettre du père biologique d'O. Kim, qui poursuit lui aussi une carrière littéraire :

À mon sens, le besoin d'écrire n'existerait pas sans cet état d'unfulfilment. [...] L'écrivain est celui qui toujours refusera de glisser cette main solitaire et vigilante sous le drap douillet. [...] Mais l'homme qui écrit ainsi n'a pas le choix. Il accepte cet état permanent d'unfulfilment et cette insomnie qui est sa nourriture. ${ }^{40}$

Partant, la création littéraire repose sur une dialectique du maître et de l'esclave : le sujet écrivant, se croyant le maître de ses idées et de son acte d'écrire, se soumet au fur et à mesure à sa motivation d'écrire, qui est cet état d'insatisfaction et qui lui dicte la vie. Notons qu'Ook Chung fait une remarque similaire à ce propos :

La création est un remède contre la solitude. On écrit pour y échapper et voilà que, quand l'œuvre est produite, on recommence. C'est une forme d'aliénation et de perversion, qui rend les écrivains infirmes à vie sur les plans émotionnel et social ${ }^{41}$.

Ainsi, pour Chung, tout écrivain, ou au moins tout écrivain " accidentel " comme lui, ressemblerait à un être qui avance en titubant sur le bord d'un abîme : son corps vacille entre l'attachement et la légèreté. La légèreté lui montre le danger de perdre pied, lui cause du désespoir, et le pousse à rompre toute complaisance existentielle ainsi qu'à mettre en mots son malaise; au contraire, l'attachement, bien que temporaire, lui promet le réconfort et la vie, une distance par rapport au malheur pour l'empêcher de se vautrer dans l'inertie. Cette dialectique créatrice est similaire à celle que propose Max Bilen dans Dialectique créatrice et structure de l'auvre littéraire. Selon Bilen, le créateur négocie sans cesse un compromis entre sa condition d'homme et sa condition de créateur : d'une part, il refuse de partager la

39 Ibid., p. 156.

$40 \quad$ Ibid., p. 236-237.

41 Micheline Lachance, "Anatomie mentale ", L'Actualité, 15 novembre 2003, p. 127. 
condition ordinaire des êtres humains en voulant être différent et devenir " autre "; d'autre part, il doit créer son univers fictif sous la forme d'un dédoublement du réel partagé. Cette dialectique est particulièrement vraie dans le cas des écrivains migrants comme Ook Chung : tout en étant membres de plusieurs communautés, ils cherchent à accéder à " autre chose " par le biais de l'héritage culturel partagé; en mêlant plusieurs codes langagiers, ils essaient de dépasser ce que les mots signifient d'ordinaire dans leur langue d'écriture - dans le cas d'Ook Chung, le français. En ce sens, l'écriture constitue une suite de nouveaux départs vers l'Autre et l'Ailleurs sans qu'aucune véritable arrivée ne soit possible pour le sujet écrivant, qui y cherche en vain un refuge et qui n'est jamais sauvé « que pour mieux [se] perdre ${ }^{42}{ }^{43}$.

Alors pour se sauver de cette malédiction de la perte et pour combler ce creux d'unfulfilment, le narrateur ressent le besoin de "désécrire les pages tristes de [s] on exil » pour revenir à l'innocence de l'enfance. Certes, le voyage au pays d'origine réalise par l'enfoncement dans le passé cette " désécriture », pour emprunter le terme de Ching Selao; cependant, la danse butô qu'il pratique au Japon développe cette même " désécriture " en travaillant la chute dans les profondeurs abyssales et la remontée résurrectionnelle pour finalement orienter la mort vers la vie. Le butô, cette "danse de l'obscurité " développée dans les années 50 au Japon, dépasse les conventions tant esthétiques qu'éthiques et guide les danseurs dans " la redécouverte des premiers gestes " humains à travers la mise en scène de la laideur (ou plutôt de la beauté négative) et des sujets interdits (tels que l'inceste, l'homosexualité, le meurtre). Suivant cette esthétique de la "virginité gestuelle", il faut se plonger dans les ténèbres abyssales pour reconquérir

42 Ook Chung, op. cit., p. 156.

43 Cet état de flottement se retrouve également dans les œuvres de Ying Chen, un autre "écrivain migrant ", qui indique dans son essai La vie probable que l'écrivain en exil est "à mi-chemin entre le réel et le probable. Il ne peut se résoudre à s'asseoir fermement sur ce qu'on appelle le réel, constamment attiré par le côté probable. Parfois il confond même les deux bouts de son chemin » (Ying Chen, Quatre mille marches, Montréal, Boréal, 2004, p. 90-91). 
l'Origine et " la création libre et individuelle », qui permet « une rupture avec les traditions et habitudes personnelles " et "une recherche constante du nouveau et du non vécu ${ }^{44}$ ». Cette chute dans les profondeurs d'origine en vue d'une ascension vers la renaissance correspond symboliquement au retour salutaire du narrateur au pays natal et au ventre maternel pour enterrer son identité morte et pour renaître sous un nouveau jour.

\section{Conclusion}

Pour conclure, la quête identitaire évoquée dans Kimchi se développe dans le cadre spatio-temporel d'une opposition entre dénaissance et renaissance, entre l'apprentissage et le désapprentissage identitaire visant à guérir le mal-être dû soit au fardeau de multiples appartenances, soit à la légèreté de la non-appartenance. Le retour spatial vers le continent natal et le retour temporel vers les profondeurs du passé s'entrecroisent pour effacer les strates identitaires mortes et en créer de nouvelles, ce qui permet l'émergence d'un imaginaire métis à la croisée des cultures. Dans le processus liant la mort à la résurrection identitaire, l'écriture (et l'art en général) remplit une fonction fondamentale : elle met l'individu en perpétuelle tension entre la légèreté du vide et l'attachement fossilisant, entre le désespoir et l'inertie. Cette dialectique créatrice correspond à un aspect fondamental des sujets migrants; en effet, ils cherchent constamment, par leur condition partagée de membre d'une ou plusieurs communautés, à dépasser ces dernières, à devenir " autre " dans cette négociation entre l'individuel et le collectif. Leur mise en cause de la frontière littéraire et leur mise en exil de la parole représentent bel et bien une forme de migrance. L'art peut lui-même prendre cette dimension transcendantale : ainsi, dans l'univers de Chung, la danse butô propose un passage vers l'accès à la renaissance identitaire par la plongée dans les profondeurs abyssales, une canalisation de la force destructrice vers la vitalité constructive. Dans cette création qui valorise les beautés " en marge ", la danse butô aide les naufragés de la vie à se réorienter, à renaître.

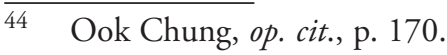




\section{Bibliographie}

Berrouët-Oriol, Robert et Robert Fournier, "L'émergence des écritures migrantes et métisses au Québec ", Québec Studies, n 14, 1992, p. 7-22.

Bhabha, Homi, Les Lieux de la culture : une théorie postcoloniale, Paris, Éditions Payot et Rivages, 2007, 414 p.

Bilen, Max, Dialectique créatrice et structure de l'œuvre littéraire, Paris, Librairie philosophique J. Vrin, 1971, 311 p.

Chen, Ying, Quatre mille marches, Montréal, Boréal, 2004, 128 p.

Chung, Ook, Kimchi, Paris, Le Serpent à plumes, 2001, 246 p.

Harel, Simon, Les Passages obligés de l'écriture migrante, Montréal, XYZ, 2005, $250 \mathrm{p}$.

Huston, Nancy, "Le Déclin de l' "identité"? ", Âmes et corps : textes choisis 1981-2003, Montréal, Leméac, 2004, 275 p.

Lachance, Micheline, "Anatomie mentale ", L'Actualité, 15 novembre 2003, p. 127.

Mouneimne-Wojtas, Tina, "L'Imaginaire "asiatique” de Ying Chen, d'Ook Chung et d'Aki Shimazaki ", dans L'Imaginaire du roman québécois contemporain : actes du colloque Université Masaryk de Brno, Brno, Masarykova univerzita Brno, Montréal, Université du Québec à Montréal, Figura, Centre de recherche sur le texte et l'imaginaire, 2006, p. 139-149.

Nadeau, Jules, "Ook Chung : la vie est un match de sumo ", La Presse, 14 septembre 2003, p. F2.

Ricœur, Paul, "L'Identité narrative ", Esprit, nos 7-8, 1988, p. 295-304.

Ricœur, Paul, Soi-même comme un autre, Paris, Seuil, 1990, 424 p.

Schützenberger, Anne Ancelin, Ä̈e, mes ä̈eux!, Paris, Desclée de Brouwer, 1993, $257 \mathrm{p}$.

Selao, Ching, "Écriture butô et altérité : Kimchi d'Ook Chung ", dans Janusz Przychodzen (dir.), Asie du soi, Asie de l'autre, Québec, Les Presses de l'Université Laval, 2009, p. 57-78.

Taylor, Charles, Les Sources du moi, Montréal, Boréal, 1998, 712 p. 